\title{
The role of TGFb in alveolar epithelial homeostatic turnover in mice
}

\author{
Mohit Aspal, Neil Vaishampayan, Nicole Jansing, Brooke Pallas, DVM, BS, and Rachel Zemans, MD* \\ Departments of Internal Medicine and Cellular and Molecular Biology, University of Michigan, Ann Arbor, 48108
}

The lung epithelium consists of alveolar Type I (ATI) and Type II (ATII) cells, with ATI cells covering the majority of the alveolar surface due to their thin, elongated shape. During homeostasis, there is slow cell turnover. ATI and ATII cells occasionally die, and the thicker, cuboidal ATII cells act as progenitor cells by proliferating and differentiating into new ATI cells. The rate of ATII cell proliferation during homeostasis is slow since ATII cells only proliferate if ATI or ATII cells perish, but exactly how slow is unknown. Moreover, the mechanisms that suppress ATII cell proliferation during homeostasis are unknown. We used unbiased stereological analysis to more accurately calculate the rate of ATII cell turnover in uninjured mice during homeostasis. Stereology refers to the three-dimensional analysis of tissue using measurements performed on two-dimensional sections, which provides more accurate measures of cell counts. We also investigated whether the transforming growth factor beta (TGFb) protein suppresses ATII cell proliferation during homeostasis. We studied the effect of the $\mathrm{TGFb}$ protein on ATII cell proliferation by using stereological analysis to compare ATII cell proliferation over a 3-month period between wild-type mice and mice with the TGFb receptor knocked out in ATII cells. The rate of ATII cell turnover during homeostasis varied among different litters of mice but had a range between 0.49-1.41\% per day. TGFb receptor knock-out mice had a nonsignificant trend towards higher total ATII cell counts after 3 months compared to wild-type mice. However, since this difference was statistically insignificant, no conclusions can currently be made about TGFb's role as a repressor of ATII cell proliferation during homeostasis. Strengthened understanding of these molecular mechanisms may ultimately lead to development of new clinical treatments that accelerate lung repair in individuals with excessive lung damage, such as patients with lung fibrosis and emphysema.

\section{Introduction}

There are two types of alveolar cells in the lung epithelium: large, flattened ATI cells that permit gas exchange and thick, cuboidal ATII cells that are the progenitor cells responsible for regenerating ATI and ATII cells during homeostasis and after injury. ${ }^{1}$ The lung epithelium serves as a barrier that protects the lungs from airborne pathogens and prevents leakage of essential epithelial fluids, such as water and blood. ${ }^{2}$ This barrier also prevents other nonepithelial fluids, such as blood, from entering and submerging the lungs, which protects the body against drowning. If there was a lot of epithelial cell death, such as during infection or after exposure to cigarette

^To whom correspondence should be addressed: zemansr@med.umich.edu smoke, the barrier function would be compromised. During homeostasis, alveolar epithelial cells have a long but limited lifespan. After the death of an occasional alveolar epithelial cell, barrier function will also be compromised unless there are adequate levels of ATII cell proliferation to replace the lost cells. Therefore, the mechanisms of ATII cell proliferation not only provide an important understanding of the lung epithelial structure, which is made up of ATI and ATII cells, but also the epithelium's function as a protective and fluid barrier and oxygen absorption for the lungs.

The lungs are relatively quiescent organs, and ATI and ATII turnover is significantly slower in the lungs than in other organs such as mammalian skin, where the epithelium rapidly turns over. ${ }^{3}$ However, in the lung, epithelial progen- 
itors are recruited primarily in response to injury (1). Still, during alveolar homeostasis, epithelial cells do turnover at a lower rate, and ATII cells play an important function as the progenitor cell, with the first step being proliferation of the ATII cell. ${ }^{4}$

To understand the mechanism behind the low homeostatic levels of ATII proliferation, we first had to accurately measure the rate of ATII cell turnover in an uninjured mouse lung during homeostasis. This rate of turnover has been calculated by researchers in the past, but the statistical methods used have been biased since the ATII cells were only counted in the $x-y$ distribution. ${ }^{5}$ By not counting cells throughout the z-distribution in a layer of lung tissue, larger ATII cells are overrepresented because they are more likely to intersect the tissue section to yield a greater series of countable events in comparison to smaller ATII cells. This results in a biased measurement of the number of ATII cells in sections of lung tissue, which in turn leads to an inaccurate calculation of the total ATII cells in the mouse lung and inaccuracies in the ATII proliferation rate. However, in our study, we used a stereological counting method in which three-dimensional interpretation of the lung tissue was performed using various two-dimensional cross-sections. This provided a more accurate, unbiased measure of the number of ATII cells in every sample frame counted.

In order to calculate the rate of ATII proliferation during homeostasis, we determined the ratio between new ATII cells formed in a 2-week period and the total ATII cell count. Once we had established a low baseline proliferation rate during homeostasis in a wild-type mouse, the next question was to understand what processes were responsible for suppressing proliferation. From previous literature, it appeared that $\mathrm{TGFb}$ played important roles in intracellular signaling which inhibited cellular proliferation. ${ }^{6}$ In one report, $\mathrm{TGFb}$ was shown to have regulatory roles as a repressor protein during tumor growth. ${ }^{7}$ Based on these past studies and our own in vitro data showing that TGFb inhibits ATII cell proliferation ${ }^{8}$, we hypothesized that TGFb suppresses ATII cell proliferation. TGFb functions by binding to its cell surface receptor and regulating cellular transcription via phosphorylation of the Smad2/Smad3 signaling system. Through this system, $\mathrm{TGFb}$ has regulatory effects on cell migration, adhesion, growth, and differentiation. ${ }^{9,10}$ Therefore, based on its signaling properties, TGFb could play an important role in regulating transcription in ATII cells, suppressing ATII cell proliferation.

To determine if TGFb suppresses ATII cell turnover during homeostasis, relative cell counts were compared between wild-type mice and ATII cell-specific TGFb receptor knock-out mice, in which ATII cells could not respond to $\mathrm{TGFb}$. Despite $\mathrm{TGFb}$ receptor knock-out mice having a trend towards higher total ATII cell counts in comparison to wild-type mice, no statistically significant correlation can currently be established. Additional experiments that statistically support TGFb's possible suppressive role move us closer towards identifying an important regulatory pathway. This knowledge could be applied in clinical treatments for lung regeneration in patients with excessive lung damage due to infection or cigarette smoke.

\section{Materials \& Methods}

\section{Animals}

The ATII cell proliferation during homeostasis experiment (EXP 1) was conducted using 6 wild-type (wt/wt) mice. Transgenic mice, in which all of the ATII cells expressed Green Fluorescent Protein (GFP), were used for every experiment. Mice in EXP 1 had an EdU pump surgically implanted in the back for 2 weeks, which allowed EdU incorporation in proliferating ATII cells. EdU is an analog of thymidine, a DNA nucleotide. When DNA replicates during proliferation, EdU is incorporated into the DNA in the place of thymidine. Therefore, all new ATII cells that formed in this period incorporated EdU, which could be stained with Cy5 and counted under the microscope. The EdU was administered for 2 weeks, since alveolar epithelial cell turnover is so slow during homeostasis.

The experiment determining the effect of TGFb on ATII cell proliferation (EXP 2) was conducted using wild-type (wt/wt) mice and ATII cell-specific TGFb receptor knockout mice (SftpcCreERT2; mTmG; TGFbRII floxed mice) which had the TGFb receptor gene knocked out from ATII cells. 4 different litters of mice were studied, each with both knock-out (flox/flox) and wild-type (wt/wt or flox/wt) subjects. Mouse 2300-1 was the only mouse with a heterogeneous (flox/wt) genotype, but since one copy of the gene is probably enough for normal expression of the TGFb receptor, Mouse 2300-1 was combined with the other wild-type mice for the purposes of statistical analysis. No EdU pump was placed in mice from EXP 2. The experiment confirming that TGFb signaling was active in ATII cells (EXP 3) was conducted using $6 \mathrm{wt} / \mathrm{wt}$ mice, all with healthy lungs. 


\section{Tissue Preparation}

The tissue was prepared as previously described. ${ }^{11}$ After euthanasia, lungs were fixed in $4 \%$ paraformaldehyde at $25 \mathrm{~cm}$ $\mathrm{H}_{2} \mathrm{O}$ inflation pressure, processed using standard techniques, and embedded in paraffin. The tissue was cut into thin sections and placed on a microscope slide. For each mouse in EXP 1, a tissue layer was randomly selected and every 12th slide of tissue was analyzed. For each mouse in EXP 2, a tissue layer was also randomly selected, but every 24th slide was analyzed. This ensured that all tissue samples studied were completely random. For each mouse in EXP 3, two random tissue layers were selected and contained either the $T G F b$ protein $(R \& D)$ or an isotype antibody $(R \& D)$, which lacks specificity to any target but matches the heavy and light chain class of antibody of the primary. The sampling of the lung sections has been described in detail in past literature. ${ }^{12}$

\section{Fluorescent Labeling}

To count cells using the stereological counting method, the ATII cells were stained so they could be observed under the fluorescence microscope. Fluorescent labeling of the lung tissues was performed by staining the tissues with a primary antibody that binds GFP followed by a secondary antibody containing a fluorescent label that binds the primary antibody. Since only the ATII cells express GFP, only the ATII cells will be labeled with the fluorescent label. To confirm that TGFb signaling was active in ATII cells, sections were stained with antibodies against TGFb and phosphorylated Smad2 (pSmad2). The fluorescent signal of the $\mathrm{TGFb}$ or pSmad2 protein was differentiated from an isotype control, which is a primary antibody that lacks specificity to any target. Fluorescent labeling of these lung tissues was performed by staining the tissues with a primary antibody and the complementary secondary antibody binding Red Fluorescent Protein (RFP). A kit was used to fluorescently label the EdU (Thermo Scientific). Under the EdU colored filter, ATII cells that were a product of regeneration were counted, since the EdU marker is incorporated into every new ATII cell's DNA. Under the GFP filter, all ATII cells were counted since GFP is expressed by all ATII cells. Under the RFP filter, the presence of TGFb and pSmad 2 was confirmed in all mice that expressed the TGFb protein and had active TGFb receptors.

\section{Staining}

In EXP 1, each tissue slide was stained with DAPI, GFP (Abcam), and EdU (Thermo). DAPI stains the nucleus of all cells, which helps distinguish ATII cells during microscopic counting. In EXP 2, the tissue slides were only stained with DAPI and the GFP antibodies, not EdU, since only the total ATII cell counts were being measured. In EXP 3, the tissue slides were stained with DAPI, and the TGFb (Abcam) and pSmad2 (Cell Signaling) antibodies. After the tissues were prepared on the slides, the sections were incubated in histochoice, ethanol (100\% to 30\%), and Milli-Q water, in order to deparaffinize and hydrate the tissue. For antigen retrieval, the slides were placed in a small slide container filled with 10X Dako Target Retrieval Solution diluted in DI water (1:10). This container was then placed into a rack inside a steamer $\left(90-95^{\circ} \mathrm{C}\right)$, boiled for 20 minutes, and cooled to room temperature (RT). For the EdU staining, the tissues on the slides were isolated with a Pap pen, dipped in TTBS to rinse the Dako Buffer, incubated in 3\%BSA-PBS, and then were permeabilized in $0.5 \%$ Triton- $X$ in PBS. A Click-iT reaction cocktail was prepared by combining Click-iT EdU reaction buffer, Neg (50 mL conical-4C), $\mathrm{CuSO}_{4}$, AlexaFluor 647 azide, and 10X Click-iT EdU buffer additive (1:10). The permeabilization buffer was dumped off, the slides were washed with $3 \%$ BSA-PBS, and $200 \mu \mathrm{L}$ of Click-iT EdU reaction cocktail was added, incubating for 1 hour at RT in the dark. For the GFP and RFP staining, the slides were washed with RT TTBS and blocked in $200 \mu \mathrm{L}$ of $10 \%$ Donkey Serum in TTBS for 1 hour at RT. The block was dumped off and the slides were incubated overnight $\left(4^{\circ} \mathrm{C}\right)$ in dilute primary antibody anti-GFP or anti-RFP in 10\% Donkey Serum in TTBS $(10 \mathrm{mg} / \mathrm{mL})(1: 800)$. The sections were then washed with cold TTBS and incubated for 1 hour in dilute $2^{\circ}$ antibody Cy3-conjugated or Cy5-conjugated AffiniPure Donkey anti-chicken IgG (1:200) in 10\% Donkey Serum in TTBS. Slides were then washed with RT TTBS and mounted with one drop of ProLong Diamond Antifade Mountant with DAPI, while the tissue was still wet. The tissue was then cured for 24 hours at RT in the dark and stored long-term at $4^{\circ} \mathrm{C}$ after cured.

\section{Stereological Counting}

An Olympus fluorescence microscope containing filters for the different DAPI, Cy3, and Cy5 fluorescent stains was used, allowing for individual counting of GFP and EdU 
stained ATII cells. Using the Visiopharm (VIS) computer software, unbiased stereological counting was performed to determine various ATII cell counts. Random area sampling was conducted with a constant step length of $800 \mu \mathrm{M}$. Since thin slices of lung tissue placed on the slide still have some thickness, we also counted ATII cells throughout the tissue at a set z-distribution of $10 \mu \mathrm{M}$. For every slide of tissue for each mouse in EXP 1, all cells that expressed GFP and all cells that expressed both GFP and EdU were counted. Every cell that expressed GFP was an ATII cell, so counting these cells provided a measure of the total ATII cell count in that slide of tissue. Every cell that expressed both GFP and EdU were ATII cells that were a product of proliferation in that 2-week period. Not all EdU cells were counted because some cells that were not ATII cells also expressed EdU. The non-ATII cells that are EdU+ are likely to be macrophages or fibroblasts, since we have previously shown that there are no other epithelial progenitors besides the ATII cell in the LPS model. ${ }^{11}$ The effect of macrophages on ATII proliferation and differentiation is largely unknown and beyond the scope of this manuscript. The ratio of new ATII cells formed in relation to the total ATII cell count determined the rate of ATII cell proliferation. For every slide of tissue for each mouse in EXP 2, only the cells that expressed GFP were counted. This determined total ATII cell counts in the tissue slides, which were then compared between wild-type and knock-out mice within the same litter, since our first experiment demonstrated that ATII proliferation can vary among mice litters.

Since random sampling was used at all stages of the experiments - including the selection of the lung, the selection of the tissue layers, and the area of the tissues analyzedstatistical Optical Fractionator calculations were performed to determine total ATII cell counts in the entire lung for each mouse. The Optical Fractionator calculation determines total cell counts using a constant block sampling fraction (bsf) (1 out of 2 lungs were analyzed), section sampling fraction (ssf) (every 12th or 24th tissue layer was analyzed), height sampling fraction (hsf) $(10 \mu \mathrm{M}$ of tissue from the entire $\mathrm{z}$ distribution thickness was analyzed), and area sampling fraction (asf) (a step length of $800 \mu \mathrm{M}$ determined random sample areas out of the entire area of tissue). The total ATII cell counts were used to calculate the ATII proliferation rates during homeostasis, using Formula 1 below:

Total ATII cell count in mouse $=($ sample cell counts $) \mathrm{x}$ $(1 / \mathrm{bsf}) \times(1 / \mathrm{ssf}) \times(1 / \mathrm{hsf}) \times(1 / \mathrm{asf})(1)$

\section{Statistical Analysis}

A one-sample t-test comparing mean total ATII cell counts between wild-type and $\mathrm{TGFb}$ receptor knock-out mice was performed. A p-value of $\leq 0.05$ was considered statistically significant.

\section{Fiji Quantification}

The Fiji software was used to calculate the integrated density, which is a quantification of the fluorescent pixels in the lung tissue images, for both the TGFb-positive tissues and the isotype antibody tissues in EXP 3. To differentiate between the non-specific signal from the isotype antibody and the targeted signal from the TGFb protein, the net integrated density was calculated by subtracting the integrated density of the isotype tissue images from the integrated density of the TGFb tissue images. This net integrated density is an arbitrary measure of the intensity of the TGFb signaling pathway in ATII cells.

\section{Results}

\section{Rate of ATII Proliferation during Homeostasis is Low}

Using the stereological counting method, the number of total ATII cells and the number of ATII cells that were a product of proliferation were counted in each slide of tissue. The ratio between ATII cells that were a product of proliferation and total ATII cells in each mouse for EXP 1 determined the rate of ATII proliferation for the 6 mice. This rate varied among the 2 litters, as the litter with Mice 3113, 3114, and 3115 (Litter 1) had rates between 6.3-9.1\%, while the litter with Mice 3151, 3152, and 3153 (Litter 2) had rates between 11.2-18.3\% (Fig. 1A). Daily ATII proliferation rates were calculated by dividing the total rates by 13 days, which is the duration that the EdU pumps remained in the mice. These rates were between $0.49-0.70 \%$ for Litter 1 and $0.86-1.41 \%$ for Litter 2 (Fig. 1B). Therefore, the rate of ATII cell proliferation for both litters was between the ranges of 0.49$1.41 \%$ per day. Despite the difference between proliferation rates for different mice litters, the overall range of the proliferation rates is relatively low, demonstrating the quiescent nature of epithelial lung cells. 
$\mathbf{A}$

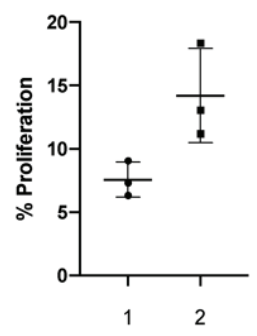

Litter
B

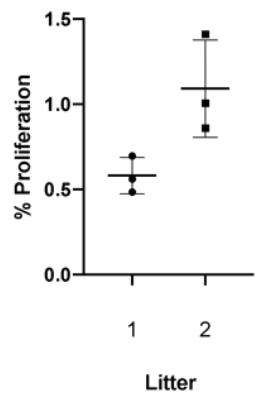

$\mathbf{A}$

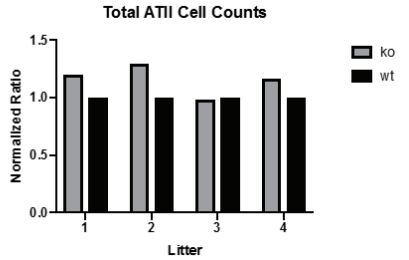

B

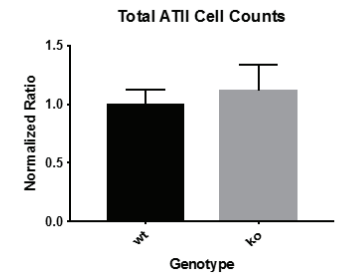

Figure 2. Total ATII cell counts are higher in TGFb receptor knock-out mice compared to wild-type mice. (A) Shows the total ATII cell counts normalized for the 4 mice litters. (B) Shows the average total ATII cell counts normalized for the combined mice litters. Total ATII cell counts for wild-type mice were rescaled to 1. Total ATII cell counts for TGFb receptor knock-out mice were typically greater than 1 .

\% ATII cell proliferation per day. There is a clear difference between proliferation rates for different mice litters, but the overall range of the proliferation rates is relatively low, demonstrating the quiescent nature of epithelial lung cells.

\section{Total ATII Cell Counts are Higher in TGFb Receptor Knock-out Mice}

In EXP 2, wild-type mice with TGFb receptors and active TGFb signaling pathways typically had lower total ATII cell counts compared to ATII cell-specific TGFb receptor knockout mice (Fig. 2A). In order to compare total ATII cell counts between litters with varying ATII proliferation rates, the total ATII cell counts for every mouse were normalized so that the average total ATII cell count for the wild-type mice rescaled to a value of 1 (Fig. 2B). A one-sample t-test comparing mean total ATII cell counts between wild-type and TGFb receptor knock-out mice was performed, where $p=0.259$, which is statistically insignificant. A power calculation was also performed, and assuming a p-value of 0.05 and a power of $80 \%$, we determined that a sample size of approximately 9 wild-type mice and $9 \mathrm{TGFb}$ receptor knock-out mice is needed to achieve statistical significance.

\section{TGFb Signaling is Active in ATII cells}

To confirm the presence of TGFb in wild-type mice in EXP 3 , the non-specific background signal from an isotype antibody control was differentiated from the specific signal from the TGFb protein (Fig. 3A). The net integrated density varied between mice, but the mean integrated densities for the TGFb-positive tissues was consistently higher than the mean integrated densities for the isotype antibody tissues,
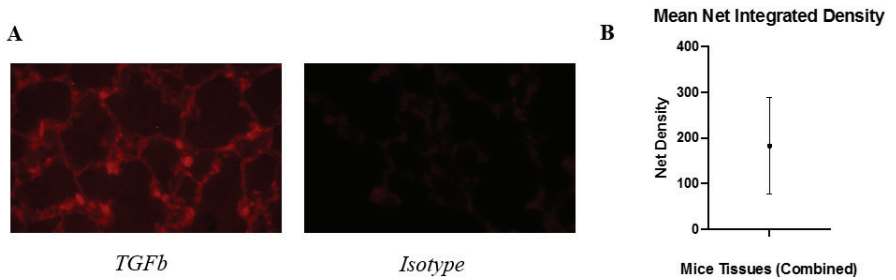

Figure 3. Fluorescence is higher in the presence of TGFb compared to an isotype antibody. (A) Displays microscopic images of TGFb-stained tissues (left) and isotype-stained tissues (right). (B) Shows the mean net integrated density combined for all tissues of every mouse. Integrated density is an arbitrary, unitless value that measures the intensity of fluorescent pixels.

resulting in a positive mean net density of 182.5 for all mice tissues combined (Fig. 3B).

\section{Discussion}

Our study used an unbiased stereological counting technique to quantify the rate of ATII proliferation during a 2-week period of lung homeostasis in wild-type mice. Additionally, using similar stereological counting, total relative ATII cell counts were compared between TGFb receptor knock-out mice and wild-type mice after a 3-month period. Consistent with our understanding that ATII cell proliferation is low, we found the rate of ATII cell proliferation to be between the ranges of $0.49-1.41 \%$ per day, varying notably between litters. This suggests that there was some unknown, biological variable inconsistent among the two litters that caused the ATII cell proliferation rate to be higher in one litter, which 
is common in animal experiments. This also indicated that the ATII cell count data collected for the other experiments needed to be compared between mice in the same litter, or the preliminary data needed to be normalized before comparing mice from different litters. Overall, our calculated ATII cell proliferation rates fall in a similar range compared to previous studies, demonstrating the relatively quiescent nature of epithelial lung cells. ${ }^{5}$ ATII cells only proliferate when ATI or ATII cells perish. The proliferation rate of ATII cells is considerably low compared to cells of other active organs, such as the skin epidermis, liver epithelium, and the gastrointestinal (GI) mucosa. ${ }^{13,14,15}$ However, ATII cells still have a higher level of proliferation compared to hippocampal neurons, which have extremely slow proliferation rates. ${ }^{16}$

In accordance with our original hypothesis, our results suggest that $\mathrm{TGFb}$ might suppress ATII cell proliferation. However, with statistically insignificant data, no definitive correlation between TGFb and suppression of ATII cell proliferation can currently be made. According to our power calculation, the statistically insignificant difference between ATII cell counts for wild-type mice and TGFb receptor knock-out mice is possibly due to an underpowered sample size. The power calculation estimated that a sample size of approximately 9 wild-type mice and 9 knock-out mice is needed to achieve statistical significance. However, even with our current sample size of 4 litters, our initial data does suggest that $\mathrm{TGFb}$ receptor knock-out mice with inactive Smad2/3 cellular transcription signaling pathways might have higher total ATII cell counts after 3 months compared to wild-type mice that experienced the regulatory effects of TGFb. TGFb's general suppressive effect is consistent with previous literature that reports the role of $\mathrm{TGFb}$ in cellular proliferation inhibition and repressed tumor growth. ${ }^{6,7}$ If further research establishes, with statistical confidence, a proven role for $\mathrm{TGFb}$ in repressing ATII cell proliferation, there would be several clinical implications. For example, there is the possibility that therapeutic suppression of TGFb signaling might promote epithelial cell repair after excessive lung damage due to infection or cigarette smoke. Additionally, drugs that increase TGFb might activate inhibitory regulatory pathways that slow down lung cancer metastasis.

We chose to analyze the lung tissue slides in EXP 1 and EXP 2 using stereology to provide accurate, unbiased measures of the ATII cell counts. However, stereological counting takes a considerably longer amount of time compared to cell counting software that only counts ATII cells in the $x-y$ distribution. Therefore, only a small number of mice subjects could be analyzed for each experiment. Our underpow- ered sample size possibly resulted in a high p-value for the experiment determining the effect of TGFb on ATII cell proliferation, leading to a statistically insignificant difference between total ATII cell counts for wild-type and knock-out mice. In the future, we will analyze the lung tissues of additional wild-type and floxed TGFb receptor mice from 2 or 3 more mice litters, which according to our power calculation appears to be sufficient in achieving statistical significance. This will expectantly strengthen our current findings and result in a statistically significant difference between total ATII cell counts for EXP 2. Another limitation of our current study was that in the ATII cell proliferation during homeostasis experiment, we assumed that ATII cells turned over only once in the 2-week period where EdU was administered. Therefore, we assumed that every EdU-stained ATII cell was a product of another ATII cell that had not proliferated within that same 2 -week period. If ATII cells did turnover more than once during the 2 weeks, our ATII cell proliferation rate may be an underestimation of the actual ATII cell proliferation rate.

This investigation establishes the foundation for further studies in this field. Using our understanding of the mechanisms behind ATII cell proliferation during homeostasis, similar studies can be devised to understand the mechanisms driving the differentiation of ATII cells into ATI cells during homeostasis, which is the second step in ATI cell turnover. Additionally, the mechanisms of lung repair during ATII cell proliferation and differentiation after injury could also be studied. TGFb does appear to suppress ATII cell proliferation during homeostasis, but its effects during differentiation or after lung injury are not well known. Moreover, the knowledge from this study can be used to create a lung cancer model, where the TGFb receptors on the cancer cells are knocked-out and any growth in the cancer is measured.

\section{References}

1. Zacharias, W. J., Frank, D. B., Zepp, J. A., Morley, M. P., Alkhaleel, F. A., Kong, J., . . Morrisey, E. E. (2018). Regeneration of the lung alveolus by an evolutionarily conserved epithelial progenitor. Nature, 555(7695), 251-255. https://doi.org/10.1038/nature25786

2. Kalinin, A. E., Kajava, A. V., \& Steinert, P. M. (2002). Epithelial barrier function: assembly and structural features of the cornified cell envelope. BioEssays: News and Reviews in Molecular, Cellular and Developmental Biology, 24(9), 789-800. https://doi.org/10.1002/ bies. 10144 
3. Gillespie, S. R., \& Owens, D. M. (2018). Isolation and Characterization of Cutaneous Epithelial Stem Cells. Methods in Molecular Biology (Clifton, N.J.). https:// doi.org/10.1007/7651_2018_171

4. Fujino, N., Kubo, H., Suzuki, T., Ota, C., Hegab, A. E., He, M., . . . Yamaya, M. (2011). Isolation of alveolar epithelial type II progenitor cells from adult human lungs. Laboratory Investigation; a Journal of Technical Methods and Pathology, 91(3), 363-378. https://doi. org/10.1038/labinvest.2010.187

5. Uhal, B. D. (1997). Cell cycle kinetics in the alveolar epithelium. American Journal of PhysiologyLung Cellular and Molecular Physiology, 272(6), L1031-L1045. https://doi.org/10.1152/ ajplung.1997.272.6.L1031

6. Blobe, G. C., Schiemann, W. P., \& Lodish, H. F. (2000). Role of transforming growth factor beta in human disease. The New England Journal of Medicine, 342(18), 1350 1358. https://doi.org/10.1056/NEJM200005043421807

7. Tang, B., Böttinger, E. P., Jakowlew, S. B., Bagnall, K. M., Mariano, J., Anver, M. R., . . . Wakefield, L. M. (1998). Transforming growth factor-beta1 is a new form of tumor suppressor with true haploid insufficiency. $\mathrm{Na}$ ture Medicine, 4(7), 802-807.

8. Riemondy, K. A., Jansing, N. L., Jiang, P., Redente, E. F., Gillen, A. E., Fu, R., . . Zemans, R. L. (n.d.). Transforming Growth Factor $\beta$ Regulates Alveolar Regeneration after LPS-Induced Lung Injury. Manuscript Submitted for Publication. Retrieved November 24, 2018.

9. Massagué, J., \& Wotton, D. (2000). Transcriptional control by the TGF-beta/Smad signaling system. The EMBO Journal, 19(8), 1745-1754. https://doi.org/10.1093/emboj/19.8.1745

10. Owen, C. R., Yuan, L., \& Basson, M. D. (2008). Smad3 knockout mice exhibit impaired intestinal mucosal healing. Laboratory Investigation; a Journal of Technical Methods and Pathology, 88(10), 1101-1109. https://doi. org/10.1038/labinvest.2008.77

11. Jansing, N. L., McClendon, J., Henson, P. M., Tuder, R. M., Hyde, D. M., \& Zemans, R. L. (2017). Unbiased Quantitation of Alveolar Type II to Alveolar Type I Cell Transdifferentiation during Repair after Lung Injury in Mice. American Journal of Respiratory Cell and Molecular Biology, 57(5), 519-526. https://doi.org/10.1165/ remb.2017-0037MA
12. Jansing, N. L., Patel, N., McClendon, J., Redente, E. F., Henson, P. M., Tuder, R. M., . . Zemans, R. L. (2018). Flow Cytometry Underestimates and Planimetry Overestimates Alveolar Epithelial Type 2 Cell Expansion after Lung Injury. American Journal of Respiratory and Critical Care Medicine, 198(3), 390-392. https://doi. org/10.1164/rccm.201709-1838LE

13. Alberts, B., Johnson, A., Lewis, J., Raff, M., Roberts, K., \& Walter, P. (2002). Extracellular Control of Cell Division, Cell Growth, and Apoptosis. Molecular Biology of the Cell. 4th Edition. Retrieved from https://www. ncbi.nlm.nih.gov/books/NBK26877/

14. Blanpain, C., \& Fuchs, E. (2009). Epidermal homeostasis: a balancing act of stem cells in the skin. Nature Reviews. Molecular Cell Biology, 10(3), 207-217. https:// doi.org/10.1038/nrm2636

15. Marongiu, F., Serra, M. P., Sini, M., Marongiu, M., Contini, A., \& Laconi, E. (2014). Cell turnover in the repopulated rat liver: distinct lineages for hepatocytes and the biliary epithelium. Cell and Tissue Research, 356(2), 333-340. https://doi.org/10.1007/s00441-014-1800-5

16. Wong, W. M., \& Wright, N. A. (1999). Cell proliferation in gastrointestinal mucosa. Journal of Clinical Pathology, 52(5), 321-333.

17. Spalding, K. L., Bergmann, O., Alkass, K., Bernard, S., Salehpour, M., Huttner, H. B., . . . Frisén, J. (2013). Dynamics of hippocampal neurogenesis in adult humans. Cell, 153(6), 1219-1227. https://doi.org/10.1016/j. cell.2013.05.002

18. Barkauskas, C. E., Cronce, M. J., Rackley, C. R., Bowie, E. J., Keene, D. R., Stripp, B. R., . . . Hogan, B. L. M. (2013). Type 2 alveolar cells are stem cells in adult lung. The Journal of Clinical Investigation, 123(7), 30253036. https://doi.org/10.1172/JCI68782

19. Castranova, V., Rabovsky, J., Tucker, J. H., \& Miles, P. R. (1988). The alveolar type II epithelial cell: a multifunctional pneumocyte. Toxicology and Applied Pharmacology, 93(3), 472-483.

20. Chung, M.-I., \& Hogan, B. L. M. (2018). Ager-CreERT2: A New Genetic Tool for Studying Lung Alveolar Development, Homeostasis, and Repair. American Journal of Respiratory Cell and Molecular Biology. https://doi. org/10.1165/rcmb.2018-0125OC 